\title{
Optimization and Form-finding of the Heavy Cable Suspending a Deck
}

\author{
J. Liu ${ }^{1}$, C.Y. Wang ${ }^{2 *}$ \\ 1. Department of Mathematics, Michigan State University, East Lansing, MI 48824, USA \\ 2. Departments of Mathematics and Mechanical Engineering, Michigan State University, East Lansing, MI \\ 48824, USA \\ E-mail: cywang@mth.msu.edu (Corresponding author)
}

Received: 25 March 2021; Accepted: 4 May 2021; Available online: 20 June 2021

\begin{abstract}
The heavy spanning cable supporting a uniform deck is important in the design of suspension bridges. The analytic design method is presented in this paper. The problem depends on three non-dimensional parameters: the ratio of cable length to the horizontal spanning distance, the ratio of vertical to horizontal distance, and the ratio of deck density to cable density. Given these parameters, useful tables of maximum tension and sag are determined. There exists an optimum cable length for which the maximum tension is minimized. In addition, it is shown that continuous loads and discrete loads are equivalent if the number of evenly-spaced discrete loads are more than 10.
\end{abstract}

Keywords: Cable; Deck; Optimization; Discrete loads.

\section{Introduction}

The heavy spanning cable, or cable with self-weight, is a classic example in physics, mathematics and engineering. It was studied since Galileo, but its exact shape, the catenary, awaits the Bernoulli brothers [1]. Recent discussions can be found in [2,3]. The catenary supporting a single concentrated load was first discussed by Routh [4]. Multiple loads were studied by Irvine and Sinclair [5], and by Kim and Lee [6] using finite elements. The catenary cable was extended to include non-uniform self-weight [7], and to three dimensions [8,9]. When inverted, the catenary represents a funicular (moment-less) arch [10].

For a suspension bridge, the main cable (which has self-weight) may also support a horizontal load such as a deck. The cable shape is no longer a catenary. In these cases, numerical means (finite elements, iteration etc) are used. See e.g. [11,12].

Recently the closed-form solution for the heavy cable loaded with a uniform deck was found independently by Wang and Wang [13] and Lewis [14]. This analytic solution greatly simplifies the computation for the cable shape, or the form-finding process. Being exact, it also serves as a benchmark for numerical and other approximate methods. Optimization was not considered in these sources.

A cable can be optimized differently, depending on the object of optimization. Aside from cost optimization (e.g. [15]), there are three basic shape optimizations. The first class of optimization considers the cable of variable cross-sectional area such that the stress everywhere is constant (fully-stressed) [16-19]. The second class of optimization considers the loaded, uniform cable which has a minimum total weight [20-22]. The third class of optimization considers the uniform cable with minimal maximum tension. Such an optimum was found by Wang [23] but only for the uniform catenary (without a load) and Wang [24] for the catenary with a single concentrated load. Minimizing total weight is different from minimizing tension. For example, the cable can be very taut, with minimal weight but very large tension.

The main purpose of the present work is to optimize the heavy uniform cable with a uniform load (deck) with respect to the least cable tension. Such an endeavor is fundamental in the design of suspension bridges. We shall consider the cable spanning different heights, which is important for sides spans of suspension bridges.

We assume the weight of the suspenders is negligible in comparison to that of the cable or the deck, and the load due to the deck is spanwise continuously distributed. The assumption of continuous distribution versus the actual situation of discrete distribution will be discussed.

\section{Formulation}

Fig. 1(a) shows a cable of weight per length $\rho$ suspending a horizontal deck of weight per length $\bar{\rho}$ through discrete suspenders of negligible weight (for twin cables, divided appropriately by two). The deck has span length 
$L$, the cable length is $l L$, and the cable ends have a height difference of $h L$. Note that the main span of a suspension bridge usually has cables of equal heights while the end spans must have different heights. Our question is, given the afore-mentioned parameters, what are the maximum tension and the sag of the cable?

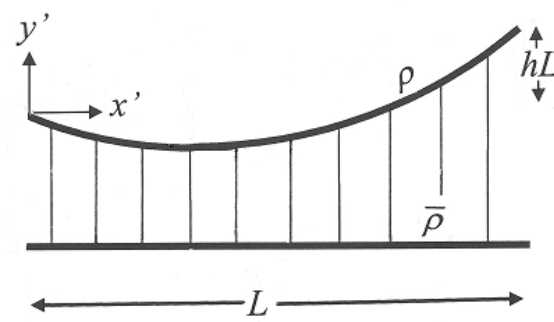

(a)

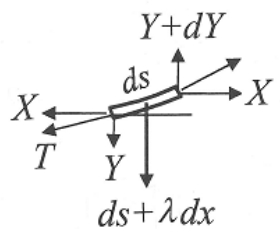

(b)

Fig. 1 (a) Spanning cable supporting a deck. (b) Force balance on an elemental segment

Normalize all lengths by $L$, and all forces by $\rho L$. Let $(x, y)$ be Cartesian coordinates place at the lower (left) end of the cable. Let $\theta$ be the local angle of inclination and $T$ be the tension of the cable. The normalized horizontal force $X$ is constant but the vertical force $Y$ varies with the arc length $s$ from the origin.

$$
X=T(s) \cos \theta=\text { constant, } Y=T(s) \sin \theta
$$

A vertical force balance on an elemental segment $d s$ (Fig. 1b) gives

$$
d Y=d s+\lambda d x
$$

where the density ratio is $\lambda=\bar{\rho} / \rho$.

Geometry dictates

$$
\frac{d x}{d s}=\cos \theta, \frac{d y}{d s}=\sin \theta
$$

Eqs.(1-3) yield

$$
\frac{d}{d s}(T \sin \theta)=1+\lambda \cos \theta
$$

or

$$
X \frac{d \theta}{d s}=\cos ^{2} \theta[1+\lambda \cos \theta]
$$

The boundary conditions are

$$
\begin{aligned}
& \left.x\right|_{s=0}=0,\left.y\right|_{s=0}=0 \\
& \left.x\right|_{s=l}=1,\left.y\right|_{s=l}=h
\end{aligned}
$$

Integrating Eqs.(3,5) gives

$$
x=X \int \frac{d \theta}{\cos \theta(1+\lambda \cos \theta)}=X[\ln (\tan \theta+\sec \theta)-F(\theta, \lambda)+A]
$$

where

$$
\begin{aligned}
& F(\theta, \lambda)=\left\{\begin{array}{c}
\frac{2 \lambda}{\sqrt{1-\lambda^{2}}} \tan ^{-1}\left(\frac{\sqrt{1-\lambda}}{\sqrt{1+\lambda}} \tan \frac{\theta}{2}\right), \lambda<1 \\
\tan \frac{\theta}{2}, \lambda=1 \\
\frac{\lambda}{\sqrt{\lambda^{2}-1}} \ln \left(\frac{\lambda+\cos \theta+\sqrt{\lambda^{2}-1} \sin \theta}{1+\lambda \cos \theta}\right), \lambda>1
\end{array}\right. \\
& y=X\left[\sec \theta-\lambda \ln \left(\frac{\sec \theta+\lambda}{1+\lambda}\right)+B\right]
\end{aligned}
$$


Except for the constants $A$ and $B$, Eqs.(8-10) essentially have the same form as the analytic solution for equal heights $[13,14]$. However, the form-finding method is different.

Our method is as follows. In order to relate $\theta$ and $s$ we further integrate Eq.(5) to obtain

$$
s=X\left[\tan \theta-\lambda \ln (\tan \theta+\sec \theta)+\lambda^{2} F(\theta, \lambda)+C\right]
$$

For the present problem, it is still not trivial to find the unknowns $A, B, C, X$ from the boundary conditions Eqs.(6.7).

Let $\theta_{0}$ be the angle at $s=0$, and $\theta_{l}$ be the angle at the end $s=l$. Evaluating Eqs. $(11,8,10)$ at the origin gives

$$
\begin{aligned}
& C=-\tan \theta_{0}+\lambda \ln \left(\tan \theta_{0}+\sec \theta_{0}\right)-\lambda^{2} F\left(\theta_{0}, \lambda\right) \\
& A=-\ln \left(\tan \theta_{0}+\sec \theta_{0}\right)+F\left(\theta_{0}, \lambda\right) \\
& B=-\sec \theta_{0}+\lambda \ln \left(\frac{\sec \theta_{0}+\lambda}{1+\lambda}\right)
\end{aligned}
$$

Eq.(11) at $s=l$ is then

$$
l=X\left[\tan \theta_{l}-\lambda \ln \left(\tan \theta_{l}+\sec \theta_{l}\right)+\lambda^{2} F\left(\theta_{l}, \lambda\right)+C\right]
$$

Then eliminate $X$ from Eqs.(7, 8, 10, 15) to yield

$$
\begin{aligned}
& l\left[\ln \left(\tan \theta_{l}+\sec \theta_{l}\right)-F\left(\theta_{l}, \lambda\right)+A\right]=\tan \theta_{l}-\lambda \ln \left(\tan \theta_{l}+\sec \theta_{l}\right)+\lambda^{2} F\left(\theta_{l}, \lambda\right)+C \\
& h\left[\ln \left(\tan \theta_{l}+\sec \theta_{l}\right)-F\left(\theta_{l}, \lambda\right)+A\right]=\sec \theta_{l}-\lambda \ln \left(\frac{\sec \theta_{l}+\lambda}{1+\lambda}\right)+B
\end{aligned}
$$

From Eqs.(16,17) we solve for $\theta_{0}, \theta_{l}$ using a 2D root search algorithm. The horizontal force $X$ can then be obtained from Eq.(15), and the shape of the cable from the parametric Eqs.(8,10). The maximum tension is at the higher end. Eq.(1) gives

$$
T=\frac{X}{\cos \theta_{l}}
$$

The sag (if any) is located at $\theta=0$ or at $s=X C$, provided $0<s<l$. Table 1 shows the results. For $\lambda=0$, the deck is absent and the cable is a free catenary. An increase in $\lambda$ increases the tension and decreases the sag. For shorter

\begin{tabular}{|c|c|c|c|c|c|c|}
\hline$I \backslash h$ & 0 & 0.25 & 0.5 & 0.75 & 1 & 1.5 \\
\hline \multirow[t]{2}{*}{1.1} & 0.8572 & 1.0940 & -- & -- & -- & -- \\
\hline & 0.199* & $0.067 *$ & & & & \\
\hline \multirow[t]{2}{*}{1.2} & 0.7621 & 0.9151 & 1.217 & -- & -- & -- \\
\hline & $0.292 *$ & $0.162 *$ & $0.027 *$ & & & \\
\hline \multirow[t]{2}{*}{1.4} & 0.7782 & 0.9098 & 1.062 & 1.286 & -- & -- \\
\hline & $0.437 *$ & $0.310 *$ & $0.177 *$ & $0.043^{*}$ & & \\
\hline \multirow[t]{2}{*}{1.6} & 0.8488 & 0.9763 & 1.111 & 1.260 & 1.467 & -- \\
\hline & $0.564 *$ & $0.437 *$ & $0.308^{*}$ & $0.174 *$ & $0.043 *$ & \\
\hline \multirow[t]{2}{*}{1.8} & 0.9342 & 1.060 & 1.190 & 1.325 & 1.474 & -- \\
\hline & $0.682 *$ & 0.556* & $0.428 *$ & $0.297 *$ & $0.164 *$ & \\
\hline \multirow[t]{2}{*}{2} & 1.027 & 1.151 & 1.279 & 1.409 & 1.544 & 1.900 \\
\hline & 0.796* & $0.670 *$ & $0.543^{*}$ & $0.414 *$ & $0.283^{*}$ & $0.024 *$ \\
\hline \multirow[t]{2}{*}{2.2} & 1.120 & 1.246 & 1.372 & 1.500 & 1.630 & 1.916 \\
\hline & 1.015* & 0.781* & $0.654^{*}$ & $0.526 *$ & 0.397* & $0.133 *$ \\
\hline \multirow[t]{2}{*}{2.4} & 1.216 & 1.342 & 1.468 & 1.594 & 1.723 & 1.989 \\
\hline & $1.015 *$ & 0.890* & $0.763 *$ & $0.636^{*}$ & $0.508^{*}$ & $0.246 *$ \\
\hline
\end{tabular}
cable lengths, the sag disappears since the cable always have positive slope. If $l<\sqrt{1+h^{2}}$ the cable cannot span the two end points. Sag always increases with the cable length $l$. The maximum tension $T$ first decreases then increases with increased $l$. Given the end points, the cable length and the densities of the cable and deck, one can obtain the tension and sag from Table 1. These tables, presented here for the first time, can be interpolated for practical purposes.

Table 1a. Maximum tension $T$ and sag $S$ (with asterisk) for given height $h$ and length of cable $l$ (density ratio $\lambda$ $=0$ ). Empty cells denote the end points cannot be spanned. 
Table 1a. (Continued).

\begin{tabular}{lllllll}
\hline 2.6 & 1.314 & 1.439 & 1.564 & 1.691 & 1.818 & 2.077 \\
& $1.122^{*}$ & $0.997^{*}$ & $0.871^{*}$ & $0.744^{*}$ & $0.617^{*}$ & $0.358^{*}$ \\
2.8 & 1.411 & 1.537 & 1.662 & 1.788 & 1.914 & 2.170 \\
& $1.228^{*}$ & $1.103^{*}$ & $0.977^{*}$ & $0.851^{*}$ & $0.724^{*}$ & $0.467^{*}$ \\
& 1.510 & 1.635 & 1.760 & 1.886 & 2.012 & 2.266 \\
& $1.334^{*}$ & $1.209^{*}$ & $1.083^{*}$ & $0.957^{*}$ & $0.830^{*}$ & $0.574^{*}$ \\
\hline
\end{tabular}

Table 1b. Maximum tension $T$ and sag $S$ (with asterisk) for given height $h$ and length of cable $l$ (density ratio $\lambda$ $=2.5$ ).

\begin{tabular}{|c|c|c|c|c|c|c|}
\hline$I M$ & 0 & 0.25 & 0.5 & 0.75 & 1 & 1.5 \\
\hline \multirow[t]{2}{*}{1.1} & 2.849 & 3.590 & -- & -- & -- & -- \\
\hline & $0.201^{*}$ & 0.069* & & & & \\
\hline \multirow[t]{2}{*}{1.2} & 2.399 & 2.814 & 3.724 & -- & -- & -- \\
\hline & $0.295 *$ & $0.165 *$ & $0.031 *$ & & & \\
\hline \multirow[t]{2}{*}{1.4} & 2.212 & 2.506 & 2.873 & 3.489 & -- & -- \\
\hline & $0.444 *$ & $0.317 *$ & $0.185 *$ & $0.052 *$ & & \\
\hline \multirow[t]{2}{*}{1.6} & 2.212 & 2.463 & 2.742 & 3.083 & 3.622 & -- \\
\hline & $0.573 *$ & $0.447 *$ & $0.318^{*}$ & $0.186^{*}$ & $0.054 *$ & \\
\hline \multirow[t]{2}{*}{1.8} & 2.263 & 2.490 & 2.730 & 2.999 & 3.326 & -- \\
\hline & $0.694 *$ & 0.568* & $0.440 *$ & $0.311^{*}$ & $0.180 *$ & \\
\hline \multirow[t]{2}{*}{2} & 2.334 & 2.545 & 2.764 & 2.998 & 3.259 & 4.095 \\
\hline & $0.810^{*}$ & $0.684^{*}$ & $0.557^{*}$ & $0.429 *$ & 0.299* & $0.039 *$ \\
\hline \multirow[t]{2}{*}{2.2} & 2.416 & 2.615 & 2.819 & 3.033 & 3.262 & 3.842 \\
\hline & $0.922 *$ & 0.797* & $0.671^{*}$ & $0.543 *$ & $0.414^{*}$ & $0.154 *$ \\
\hline \multirow[t]{2}{*}{2.4} & 2.503 & 2.693 & 2.887 & 3.087 & 3.297 & 3.782 \\
\hline & $1.033 *$ & 0.907* & $0.781^{*}$ & $0.654 *$ & $0.527 *$ & $0.268 *$ \\
\hline \multirow[t]{2}{*}{2.6} & 2.594 & 2.777 & 2.963 & 3.153 & 3.350 & 3.784 \\
\hline & $1.141 *$ & $1.016 *$ & $0.890 *$ & $0.763 *$ & 0.636* & $0.380 *$ \\
\hline \multirow[t]{2}{*}{2.8} & 2.687 & 2.864 & 3.044 & 3.226 & 3.414 & 3.816 \\
\hline & $1.248^{*}$ & $1.123^{*}$ & 0.997* & $0.871 *$ & $0.745^{*}$ & $0.489 *$ \\
\hline \multirow[t]{2}{*}{3} & 2.782 & 2.954 & 3.128 & 3.305 & 3.485 & 3.865 \\
\hline & $1.355 *$ & $1.230 *$ & $1.104 *$ & $0.978 *$ & $0.852 *$ & $0.597 *$ \\
\hline
\end{tabular}

Table 1c. Maximum tension $T$ and sag $S$ (with asterisk) for given height $h$ and length of cable $l$ (density ratio $\lambda$ $=5$ )

\begin{tabular}{|c|c|c|c|c|c|c|}
\hline$I \backslash h$ & 0 & 0.25 & 0.5 & 0.75 & 1 & 1.5 \\
\hline \multirow[t]{2}{*}{1.1} & 4.849 & 6.088 & -- & -- & -- & -- \\
\hline & $0.201 *$ & $0.069 *$ & & & & \\
\hline \multirow[t]{2}{*}{1.2} & 4.031 & 4.709 & 6.208 & -- & -- & -- \\
\hline & $0.296 *$ & $0.167 *$ & $0.033^{*}$ & & & \\
\hline \multirow[t]{2}{*}{1.4} & 3.644 & 4.102 & 4.687 & 5.681 & -- & -- \\
\hline & $0.446 *$ & 0.319* & $0.188^{*}$ & $0.054 *$ & & \\
\hline \multirow[t]{2}{*}{1.6} & 3.574 & 3.953 & 4.379 & 4.912 & 5.772 & -- \\
\hline & $0.575 *$ & $0.449 *$ & $0.321^{*}$ & $0.190 *$ & 0.058* & \\
\hline \multirow[t]{2}{*}{1.8} & 3.591 & 3.924 & 4.280 & 4.684 & 5.189 & -- \\
\hline & $0.697 *$ & $0.571 *$ & $0.444^{*}$ & $0.315^{*}$ & 0.183* & \\
\hline \multirow[t]{2}{*}{2} & 3.642 & 3.944 & 4.259 & 4.601 & 4.991 & 6.294 \\
\hline & $0.813 *$ & $0.687 *$ & $0.561^{*}$ & $0.433^{*}$ & $0.304^{*}$ & $0.043 *$ \\
\hline \multirow[t]{2}{*}{2.2} & 3.711 & 3.990 & 4.277 & 4.582 & 4.914 & 5.786 \\
\hline & 0.926* & 0.801* & $0.675^{*}$ & $0.547 *$ & $0.419 *$ & $0.158 *$ \\
\hline \multirow[t]{2}{*}{2.4} & 3.789 & 4.050 & 4.318 & 4.597 & 4.893 & 5.599 \\
\hline & $1.037 *$ & 0.911* & $0.785^{*}$ & 0.659* & $0.531 *$ & $0.273 *$ \\
\hline \multirow[t]{2}{*}{2.6} & 3.874 & 4.121 & 4.373 & 4.632 & 4.904 & 5.519 \\
\hline & $1.146 *$ & $1.020 *$ & 0.895* & 0.768* & $0.641 *$ & $0.385 *$ \\
\hline \multirow[t]{2}{*}{2.8} & 3.962 & 4.198 & 4.437 & 4.682 & 4.936 & 5.492 \\
\hline & $1.254 *$ & 1.128* & $1.002 *$ & $0.876^{*}$ & $0.750 *$ & $0.494 *$ \\
\hline \multirow[t]{2}{*}{3} & 4.053 & 4.280 & 4.508 & 4.742 & 4.982 & 5.497 \\
\hline & $1.360 *$ & $1.235 *$ & $1.109 *$ & 0.983* & $0.857 *$ & $0.603 *$ \\
\hline
\end{tabular}




\section{Optimization}

Since the maximum tension is large when the cable is taut (large horizontal force $X$ ), and again large when the cable is slack (large self-weight), there exists an optimum length for given end points and relative deck weight such that maximum tension is minimized. Using our method described in the previous section and varying the cable length, this optimum is determined. Table 2 shows the optimum cable length for various deck relative weights $\lambda$ and height ratios $h$. The optimum length (for minimum tension) increases with both deck weight and height difference.

Table 2. The optimum length $l$, the maximum tension $T$ (in parentheses), and the sag $S$ (in brackets).

\begin{tabular}{llllll}
\hline$h \backslash \lambda$ & 0 & 0.5 & 1 & 2 & 5 \\
\hline 0 & 1.258 & 1.319 & 1.370 & 1.453 & 1.637 \\
& $(0.7544)$ & $(1.057)$ & $(1.351)$ & $(1.922)$ & $(3.573)$ \\
0.5 & {$[0.338]$} & {$[0.383]$} & {$[0.421]$} & {$[0.479]$} & {$[0.598]$} \\
& 1.377 & 1.461 & 1.537 & 1.670 & 1.987 \\
& $(1.061)$ & $(1.417)$ & $(1.757)$ & $(2.410)$ & $(4.259)$ \\
1 & {$[0.161]$} & {$[0.223]$} & {$[0.275]$} & {$[0.361]$} & {$[0.553]$} \\
& 1.673 & 1.762 & 1.846 & 2.007 & 2.414 \\
& $(1.453)$ & $(1.839)$ & $(2.208)$ & $(2.914)$ & $(4.893)$ \\
2 & {$[0.0859]$} & {$[0.148]$} & {$[0.203]$} & {$[0.302]$} & {$[0.539]$} \\
& 2.491 & 2.571 & 2.656 & 2.828 & 3.320 \\
& $(2.351)$ & $(2.764)$ & $(3.161)$ & $(3.925)$ & $(6.066)$ \\
5 & {$[0.0387]$} & {$[0.0896]$} & {$[0.142]$} & {$[0.244]$} & {$[0.519]$} \\
& 5.335 & 5.395 & 5.464 & 5.620 & 6.143 \\
& $(5.258)$ & $(5.691)$ & $(6.116)$ & $(6.944)$ & $(9.302)$ \\
& {$[0.0138]$} & {$[0.0492]$} & {$[0.0910]$} & {$[0.181]$} & {$[0.466]$} \\
\hline
\end{tabular}

The $\lambda=0$ (cable without a deck) values agree, to $1 \%$, with the results of the free catenary studied by Wang [23]. Fig.2 shows typical shapes of the optimum cable.

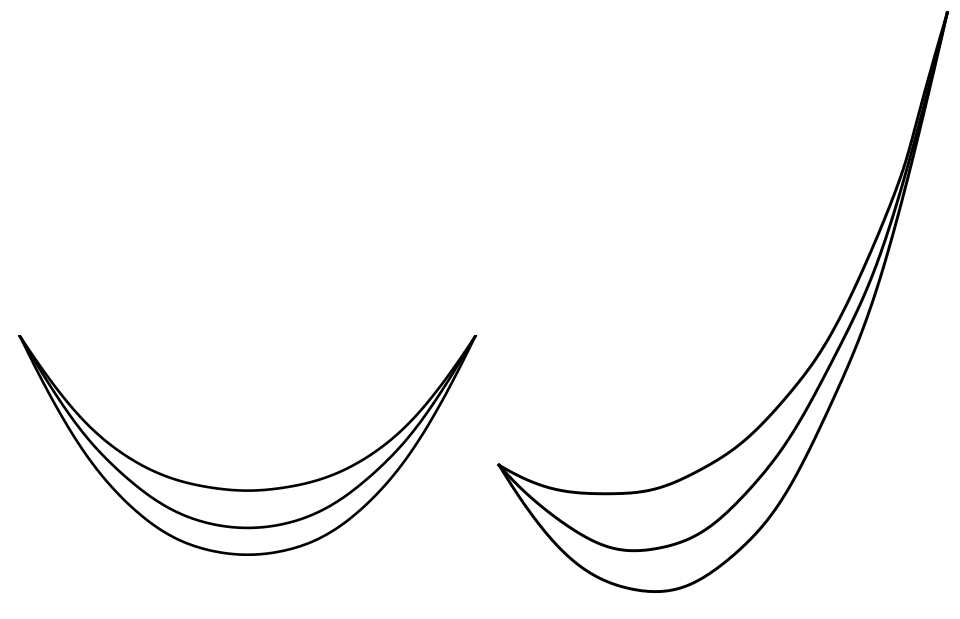

(a)

(b)

Fig. 2. Optimum cable shapes. The cable is supported at two ends with a deck (not shown). The horizontal distance between the supports is $L$ while the vertical height difference is $h L$. The ratio of the deck density to the cable density is $\lambda$. (a) $h=0$, from top: $\lambda=0,1,2$ (b) $h=1$, from top: $\lambda=0,1,2$

\section{Discrete loads}

In the previous formulation we assumed the deck weight is continuously distributed on the cable. However, for a suspension bridge the deck is usually hung from the cable at equally-spaced discrete points. In this section we investigate the condition such that the continuous load would be a good approximation to the discrete load. We shall illustrate using a cable which spans equal heights $(h=0)$. 


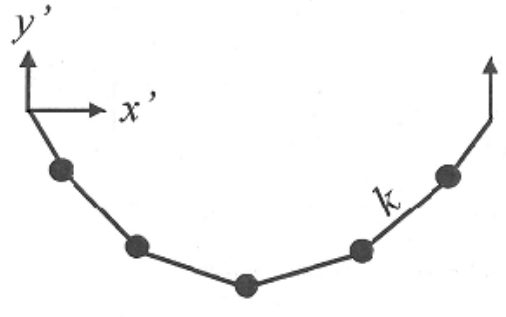

(a)

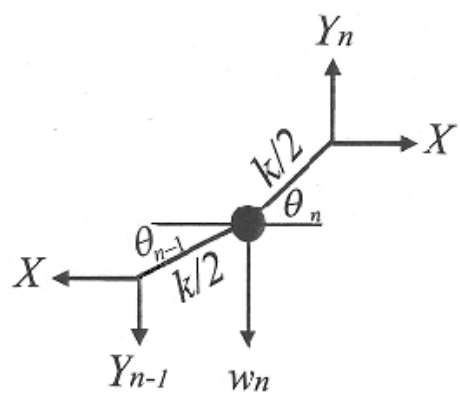

(b)

Fig. 3. (a) Hanging a string of weights (b) Force balance on the $n$th weight.

Fig. 3(a) shows a heavy cable discretized into $N$ concentrated weights connected by cable segments of negligible mass. The lengths and forces are normalized as before. The total length of the cable is $l=N k$, where $k$ is the distance between the weights, except the distance to the ends is $k / 2$. Fig. 3(b) shows the force balance on the $n^{\text {th }}$ weight from the left end. Let the location of this weight be $\left(x_{n}, y_{n}\right)$ and the inclination angles of the cable segments be $\theta_{n-1}$ and $\theta_{n}$ as shown. The discrete weight includes the normalized self-weight of the cable $k$ and the weight of the deck, proportional to the vertical projection

$$
w_{n}=k+k \lambda\left(\cos \theta_{n-1} / 2+\cos \theta_{n} / 2\right)
$$

The vertical force balance is

$$
Y_{n}=w_{n}+Y_{n-1}
$$

The constant horizontal force $X$ is related by

$X \tan \theta_{n}=Y_{n}$

Eqs.(19-21) yield

$$
X\left(\tan \theta_{n}-\tan \theta_{n-1}\right)=k+k \lambda\left(\cos \theta_{n}+\cos \theta_{n-1}\right) / 2
$$

From geometry, the coordinates are

$$
\begin{aligned}
& x_{n}=x_{n-1}+\frac{k}{2}\left(\cos \theta_{n}+\cos \theta_{n-1}\right) \\
& y_{n}=y_{n-1}+\frac{k}{2}\left(\sin \theta_{n}+\sin \theta_{n-1}\right)
\end{aligned}
$$

Due to symmetry

$$
\theta_{n}=-\theta_{N-n}
$$

the vertical forces at the ends are equal to half the total weight

$$
-Y_{0}=Y_{N}=\frac{k N+\lambda}{2}
$$

From Eqs. $(21,26)$

$$
\theta_{0}=-\tan ^{-1}\left(\frac{k N+\lambda}{2 X}\right)
$$

Our method of solution is as follows. Given the deck density ratio $\lambda$, the number of concentrated weights $N$, the length of cable $l$, then segment length is $k=l / N$. Now guess $X$ and use Eq.(27) as an initial condition for the nonlinear first order difference equation Eq.(22). When the sequence of $\theta_{n}$ is obtained, Eq.(23) yields 


$$
x_{n}=\frac{k}{2}\left(\cos \theta_{0}+2 \sum_{j=1}^{n-1} \cos \theta_{j}\right)
$$

Thus if

$$
x_{N}+\frac{k}{2} \cos \theta_{N}=1
$$

a solution is obtained. Otherwise the guess $X$ is adjusted. Then the maximum tension is

$$
T=\sqrt{Y_{0}^{2}+X^{2}}
$$

Eq.(24) gives the vertical displacement. For the $n^{\text {th }}$ weight

$$
y_{n}=\frac{k}{2}\left(\sin \theta_{0}+2 \sum_{j=1}^{n-1} \sin \theta_{j}\right)
$$

If $N$ is even, $\theta_{N / 2}=0$ and the cable segment at the mid point is horizontal. The sag is

$$
S=-y_{N / 2}=-y_{1+N / 2}
$$

If $N$ is odd, the mid point is at the weight at $n=(N+1) / 2$. Thus the sag is

$$
S=-y_{(N+1) / 2}
$$

Our question is, for the same length and weight of the cable and the weight of the deck, when would the discrete solution of this section be equivalent to the continuous solution of the previous section? Since $l$ is also the normalized total weight of the cable, we fix $l=N k$ and increase $N$ (decrease $k$ ), starting from a single concentrated weight $N=1$.

Table 3 shows the results when the deck is absent $(\lambda=0)$ and $l=2$. Notice when odd $N$ increases, the horizontal force $X$, the maximum tension $T$ and the sag $S$ decrease to the continuous values indicated by $N=\infty$. When even $N$ increases, $X, T, S$ increase to the continuous values. The table shows that when $N>10$, the maximum tension is within $0.1 \%$ and the sag is within $1 \%$ of the continuous values. We conclude that a continuous catenary cable is statically equivalent to a string of equally-spaced discrete weights if the number of weights is more than 10 .

Table 4 shows the case when the cable is supporting a deck. Here we used a density ratio of $\lambda=5$ and the optimum length of $l=1.637$ from Table 2 . Although the tension is larger and the sag smaller, the general trend with increased $N$ is similar to that of Table 3, i.e., for $N>10$ the cable with discrete loads is equivalent to the cable with continuous loads.

Table 3. Results for the horizontal force $X$, the maximum tension $T$ and the sag $S$ for equal heights and $\lambda=0, l=$ 2. The cable is approximated by $N$ discrete weights.

\begin{tabular}{lccc}
\hline$N$ & $X$ & $T$ & $S$ \\
\hline 1 & 0.577 & 1.155 & 0.866 \\
2 & 0.000 & 1.000 & 0.500 \\
3 & 0.265 & 1.035 & 0.844 \\
4 & 0.215 & 1.023 & 0.704 \\
5 & 0.238 & 1.028 & 0.824 \\
6 & 0.228 & 1.026 & 0.753 \\
7 & 0.232 & 1.027 & 0.812 \\
8 & 0.230 & 1.026 & 0.772 \\
9 & 0.231 & 1.026 & 0.806 \\
10 & 0.230 & \multicolumn{2}{c}{0.781} \\
11 & 0.230 & & 0.803 \\
12 & & & 0.786 \\
13 & & & 0.801 \\
14 & & & 0.789 \\
$\infty$ & 0.230 & 1.026 & 0.796 \\
\hline
\end{tabular}


Table 4. Results for the horizontal force $X$, the maximum tension $T$ and the sag $S$ for equal heights and $\lambda=5, l=$ 1.637. The cable is approximated by $N$ discrete weights.

\begin{tabular}{lccc}
\hline$N$ & $X$ & $T$ & $S$ \\
\hline 1 & 2.561 & 4.192 & 0.648 \\
2 & 0.755 & 3.403 & 0.399 \\
3 & 1.346 & 3.581 & 0.627 \\
4 & 1.281 & 3.557 & 0.535 \\
5 & 1.321 & 3.572 & 0.613 \\
6 & 1.314 & 3.569 & 0.568 \\
7 & 1.321 & 3.572 & 0.606 \\
8 & 1.320 & 3.572 & 0.581 \\
9 & 1.322 & 3.572 & 0.603 \\
10 & 1.322 & 3.572 & 0.587 \\
11 & 1.323 & 3.573 & 0.602 \\
12 & 1.323 & 3.573 & 0.591 \\
13 & 1.324 & \multicolumn{2}{c}{0.601} \\
14 & & 3.573 & 0.593 \\
$\infty$ & 1.326 & & 0.598 \\
\hline
\end{tabular}

\section{Conclusions}

The form-finding method is as follows. Given the horizontal span $L$, the weight per length of the cable $\rho$ the weight per length of the deck $\bar{\rho}$, the height between the cable ends $h L$, and the cable length $l L$, one can find the maximum tension $T^{\prime}=T \rho L$ and sag $S L$ form Table 1 . However, if one seeks optimum length such that the maximum tension is minimized, then one uses Table 2 . Notice that Tables 1,2 are applicable to systems with more than 10 discrete suspenders. If there are fewer suspenders, the discrete load method of Section 4 should be used.

The spanning cable with non-negligible weight and suspending a uniform deck is now solved semi-analytically. The problem is determined by three non-dimensional parameters: the cable length ratio $l$, the height ratio $h$, and the density ratio $\lambda$. Useful tables for maximum tension and sag are constructed. In addition, optimum cables lengths for minimum tension are found. These new results are useful for the preliminary design of deck-carrying cables, such as suspension bridges.

\section{Notation}

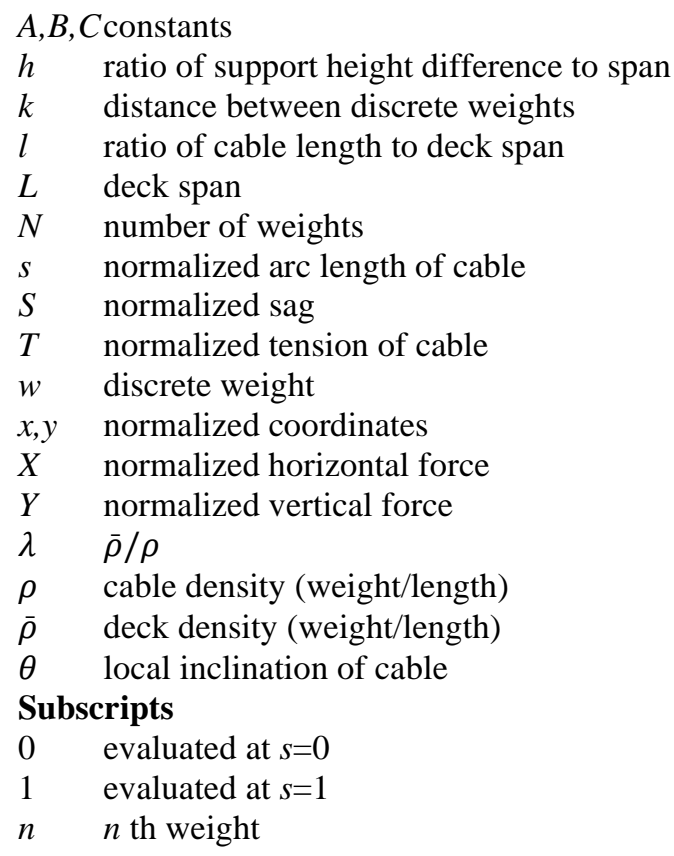




\section{References}

[1] Salmon G. Higher plane curves. Chelsea, New York. 1960; p.289.

[2] Griva IA. Case studies in optimization: Catenary problem. Opt. Eng. 2009; 6: 463-482.

[3] Behroozi F. A fresh look at the catenary. Eur. J. Phys. 2014; 35: 055007.

[4] Routh EJ. A treatise on analytical statics. Vol.1 $2^{\text {nd }}$ Ed. 1909; Chap. 10.

[5] Irvine HM, Sinclair GB. The suspended elastic cable under the action of concentrated vertical loads. Int. J. Solids Struct. 1976; 12: 309-317.

[6] Kim KS, Lee HS. Analysis of target configurations under dead loads for cable-supported bridges. Comp. Struct. 2001; 79: 2681-2692.

[7] Fallis MC. Hanging shapes of nonuniform cables. Am. J. Phys.1997; 65: 117-122.

[8] Impollonia N, Ricciardi G, Saitta F. Statics of elastic cables under 3D point forces. Int. J. Solids Struct. 2011; 48: $1268-1276$.

[9] Greco L, Impollonia N, Cuomo M. A procedure for the static analysis of cable structures following elastic catenary theory. Int. J. Solids Struct. 2014; 51: 1521-1533.

[10] Heyman J. The masonry arch. Horwood, Chichester, England 1982.

[11] Li C, He J, Zhang Z, Liu Y, Ke H, Dong C, Li H. An improved analytical algorithm on main cable system of suspension bridge. Appl. Sci. 2018; 8:1358.

[12] Zhou Y, Chen S. Iterative nonlinear cable shape and force finding technique of suspension bridges using elastic catenary configuration. J. Eng. Mech. 2019;145(5): 04019031.

[13] Wang CY, Wang CM. Closed-form solutions for funicular cables and arches. Acta Mech. 2015; 226:16411645.

[14] Lewis WJ. Mathematical model of a moment-less arch. Proc. Roy. Soc. A. 2016; 472: 20160019.

[15] Lewis WJ. A mathematical model for assessment of material requirements for cable supported bridges: Implications for conceptual design. Eng. Struct. 2012; 42: 266-277.

[16] Hill RD, Rozvany GIN, Wang CM, Leong KH. Optimization, spanning capacity, and cost sensitivity of fullystressed arches. J. Struct. Mech. 1979; 7: 375-410.

[17] Tadjbakhsh IG. Stability and optimum design of arch-type structures. Int. J. Solids Struct. 1981; 17: 565-574.

[18] Serra M. Optimal arch: Approximate analytical and numerical solutions. Comp. Struct. 1994; 52: 1213-1220.

[19] Marano GC, Trentadue F, Petrone F. Optimal arch shape solution under static vertical loads. Acta Mech. 2014; 225: 679-686.

[20] Wang CM. Optimum shape of cables. J. Eng. Mech. 1984; 110: 1649-1653.

[21] Teo KL, Ang BW, Wang CM. Least weight cables: Optimal parameter selection approach. Eng. Opt. 1986; 9: 249-264.

[22] Wang CM, Pulmano VA, Lee SL. Cable optimization under self-weight and concentrated loads. J. Struct. Mech. 1986; 14: 191-207.

[23] Wang CY. The optimum spanning catenary. Eur. J. Phys. 2015; 36: 028001.

[24] Wang CY. Optimum length of a heavy cable with a concentrated load. J. Eng. Mech. 2017; 143(12): 06017017.

(C) 2021 by the author(s). This work is licensed under a Creative Commons Attribution 4.0 International License (http://creativecommons.org/licenses/by/4.0/). Authors retain copyright of their work, with first publication rights granted to Tech Reviews Ltd. 